\title{
PASK Gene
}

National Cancer Institute

\section{Source}

National Cancer Institute. PASK Gene. NCI Thesaurus. Code C132043.

This gene is involved in energy homeostasis. 